\title{
Twisted mass, overlap and Creutz fermions: Cut-off effects at tree-level of perturbation theory
}

\author{
K. Cichy a , J. González López ${ }^{\text {b,c,* }}$, K. Jansen ${ }^{\text {c }}$, A. Kujawa a , A. Shindler ${ }^{\text {d }}$ \\ a Adam Mickiewicz University of Poznan, Faculty of Physics, Umultowska 85, 61-614 Poznan, Poland \\ ${ }^{\mathrm{b}}$ Humboldt-Universität zu Berlin, Institut für Physik, Newtonstrasse 15, 12489 Berlin, Germany \\ c DESY, Zeuthen, Platanenallee 6, D-15738 Zeuthen, Germany \\ d Theoretical Physics Division, Department of Mathematical Sciences, University of Liverpool, \\ Liverpool L69 7ZL, UK
}

Received 26 February 2008; accepted 5 March 2008

Available online 15 March 2008

\begin{abstract}
We study cutoff effects at tree-level of perturbation theory for maximally twisted mass Wilson, overlap and the recently proposed Creutz fermions. We demonstrate that all three kinds of lattice fermions exhibit the expected $\mathrm{O}\left(a^{2}\right)$ scaling behaviour in the lattice spacing. In addition, the sizes of these cutoff effects are comparable for the three kinds of lattice fermions considered here. Furthermore, we analyze situations when twisted mass fermions are not exactly at maximal twist and when overlap fermions are studied in comparison to twisted mass fermions when the quark masses are not matched.
\end{abstract}

(c) 2008 Elsevier B.V. All rights reserved.

\section{Introduction}

This paper is an investigation of the scaling behaviour towards the continuum limit for different kinds of lattice fermions at tree-level of perturbation theory. In a previous conference contribution [1] we had only discussed the case of Wilson twisted mass fermions [2,3]. See Ref. [4] for a review on twisted mass lattice QCD. Here we add overlap fermions [5]. See Ref. [6] for a review on chirally symmetric lattice actions and related topics. During the completion of this paper a new kind of lattice fermion appeared in the literature, the so-called Creutz fermi-

\footnotetext{
* Corresponding author at: Humboldt-Universität zu Berlin, Institut für Physik, Newtonstrasse 15, 12489 Berlin, Germany.

E-mail address: jenifer.gonzalez.lopez@ desy.de (J. González López).
} 
ons [7] and we decided to investigate also these lattice fermions, as well as the fermions defined by a related action suggested by Borici [8]. We will in the following generically refer to Creutz fermions having in mind both Creutz and Borici formulations.

All of these three kinds of lattice fermions are expected to show an $\mathrm{O}\left(a^{2}\right)$ scaling behaviour in the lattice spacing. While for overlap fermions this is achieved by an exact lattice chiral symmetry, for twisted mass fermions this is achieved by a residual $N_{f}=2$ flavoured continuum chiral symmetry which needs, however, a tuning of the (untwisted) PCAC quark mass to zero. Creutz fermions exhibit an exact $N_{\mathrm{f}}=2$ flavoured continuum chiral symmetry, but they break however a number of discrete symmetries such as parity, charge conjugation and time reflection [9]. This could lead to a plethora of terms in the Symanzik effective action, which would make approaching the continuum limit a rather difficult task.

Wilson twisted mass fermions are described by an ultra-local action with only nearest neighbour interactions. Hence, they are comparably cheap to simulate. Their major drawback is the explicit $\mathrm{O}\left(a^{2}\right)$ isospin-breaking. Overlap fermions have the great advantage of an exact lattice chiral symmetry [10]. However, while still local in the field theoretical sense [11], they couple each lattice point with all others and are an order of magnitude more expensive to simulate than twisted mass fermions. Finally, Creutz fermions [7] are also described by an ultra-local action, again with only nearest neighbour interactions. As has been shown recently, they break however a number of discrete symmetries and isospin symmetry [9].

Twisted mass and Creutz fermions are defined for $N_{f}=2$ flavours of quarks. These two quark flavours can either be taken as mass degenerate, or, some explicit flavour breaking term has to be added. In contrast, overlap fermions can be used for a single quark flavour.

The focus in this paper is to study the scaling behaviour with the lattice spacing. In particular, we are interested in the relative size of the cutoff effects comparing maximally twisted mass, overlap and Creutz lattice fermions.

In particular, we will consider the scaling behaviour of the meson correlation function the pseudoscalar mass and decay constant as well as the nucleon mass. It is important to remark here that, at tree-level of perturbation theory there is, of course, nothing like a real meson or baryon. However, since we use the same interpolating fields as in full QCD, we will take the freedom to use the notation of mesons and baryons throughout this paper.

We will also address the question of the size of cutoff effects when twisted mass fermions are not exactly tuned to maximal twist. In addition, we investigate ratios of mesonic quantities built from twisted and overlap fermions when the quark masses are unmatched.

While actual practical calculations with overlap and twisted mass fermions are already rather advanced, the formulation of chiral invariant fermions following Creutz is still very new.

In [1] we gave a first account of the size of cut-off effects at tree-level of perturbation theory for twisted mass fermions. Here we extend the techniques described in [1], following [12], to the case of overlap and Creutz fermions. A more detailed account of our earlier calculations and a pedagogical introduction to the techniques we have used, can be found in [13].

\section{Lattice propagators}

In this section we provide the momentum space propagators for the different kinds of lattice fermions we have considered. They are the building blocks for the computation of the meson and nucleon correlation functions from which in turn the masses and decay constants are extracted. To fix the notation our setup is a hypercubic lattice of size $L^{3} \times T$ with spacing $a$. 


\section{Wilson twisted mass fermions}

The expression for the Wilson twisted mass (Wtm) fermion propagator in the twisted basis, at tree-level of perturbation theory (PT) and in momentum space can be derived from the Wtm operator given for example in Ref. [4], and it is given by

$$
\tilde{S}_{\mathrm{Wtm}}(p)=\frac{-i \stackrel{\circ}{p}_{\mu} \gamma_{\mu} \mathbb{1}_{f}+M(p) \mathbb{1}_{f}-i \mu_{q} \gamma_{5} \tau_{3}}{\sum_{\mu} \stackrel{\circ}{p}_{\mu}^{2}+M(p)^{2}+\mu_{q}^{2}}
$$

where

$$
\stackrel{\circ}{p}_{\mu}=\frac{1}{a} \sin \left(a p_{\mu}\right), \quad \hat{p}_{\mu}=\frac{2}{a} \sin \left(\frac{a p_{\mu}}{2}\right), \quad M(p)=m_{0}+\frac{a}{2} \sum_{\mu} \hat{p}_{\mu}^{2},
$$

and where $\mathbb{1}$ and $\mathbb{1}_{f}$ are the identity matrices in Dirac and flavour space. The structure in colour space has not been written since it is just an identity matrix at tree level of PT. The parameters $m_{0}$ and $\mu_{q}$ represent the untwisted and twisted quark masses, respectively. Maximal twist-in the case of tree-level of perturbation theory-is achieved by setting $m_{0}=0$. We then expect to have only $\mathrm{O}\left(a^{2}\right)$ lattice spacing effects in physical correlation functions [3].

\section{Overlap fermions}

The expression for the overlap propagator in momentum space at tree-level of perturbation theory can be derived from the expression of the overlap operator given for example in Ref. [10], and it is

$$
\tilde{S}_{\mathrm{ov}}(p)=\frac{-i\left(1-\frac{m a}{2}\right) F(p)^{-1 / 2} \stackrel{\circ}{p}_{\mu} \gamma_{\mu}+\mathcal{M}(p) \mathbb{1}}{\left(1-\frac{m a}{2}\right)^{2} F(p)^{-1} \sum_{\mu} \stackrel{\circ}{p}_{\mu}^{2}+\mathcal{M}(p)^{2}},
$$

where:

$$
\begin{aligned}
& F(p)=1+\frac{a^{4}}{2} \sum_{\mu<v} \hat{p}_{\mu}^{2} \hat{p}_{v}^{2}, \\
& \mathcal{M}(p)=\frac{1}{a}\left(1+\frac{m a}{2}-\left(1-\frac{m a}{2}\right) F(p)^{-1 / 2}\left(1-\frac{a^{2}}{2} \sum_{\mu} \hat{p}_{\mu}^{2}\right)\right)
\end{aligned}
$$

and $\mathbb{1}$ is the identity matrix in Dirac space. Note that in the case of overlap fermions we only discuss one flavour. Due to the existence of an exact lattice chiral symmetry, we again expect an $\mathrm{O}\left(a^{2}\right)$ scaling behaviour towards the continuum limit, if the correlation functions are computed with the proper improved operators (see for example Ref. [14]).

\section{Creutzfermions}

The Creutz-Dirac operator can be found in different forms in Refs. [7-9]. We have rescaled the Creutz-Dirac operator with a factor $R$ that we leave unspecified for the moment. As will be discussed below and in Appendix A, this normalization factor $R$ is needed to obtain at tree-level the correct continuum limit for the correlation functions we have studied. In Appendix A we define all the relevant functions and we show that the Creutz operator can be brought to the form

$$
D_{C}(p)=i \sum_{\mu} \stackrel{\circ}{p}_{\mu} \bar{\gamma}_{\mu}-i \frac{a}{2} \sum_{\mu} \hat{p}_{\mu}^{2} \bar{\Gamma}_{\mu}+m_{0} \mathbb{1},
$$


where $\bar{\gamma}_{\mu}$ and $\bar{\Gamma}_{\mu}$ are linear combinations of gamma matrices defined in Appendix A. We recall that Creutz fermions, as explicitly shown in Appendix A, depend in general on two parameters $R$ and $C$. From our final expression of the Creutz operator (6), we can obtain the quark propagator for Creutz fermions. In the continuum limit, the quark propagator can be written as

$$
\tilde{S}_{C}^{\text {cont }}(p)=\frac{-i \sum_{\mu} p_{\mu} \bar{\gamma}_{\mu}+m_{0} \mathbf{1}}{\sum_{\mu} \sum_{\rho} p_{\mu} p_{\mu} \bar{a}_{\rho \mu} \bar{a}_{\rho \mu}+\sum_{\mu \neq \nu} \sum_{\rho} p_{\mu} p_{\nu} \bar{a}_{\rho \mu} \bar{a}_{\rho \nu}+m_{0}^{2}}
$$

which is not the continuum Dirac propagator unless the values of $C$ and $R$, whose dependence is hidden in the matrices $\bar{a}$ and $\bar{\gamma}_{\mu}$, are chosen properly.

From Eq. (7) it is also clear that in order to obtain the correct continuum limit of the quark propagator, we have to impose that $\bar{\gamma}=\bar{a}^{T} \gamma$ is again a gamma matrix, which is equivalent to say that the following relation holds true

$$
\left\{\left(\bar{a}^{T} \gamma\right)_{\mu},\left(\bar{a}^{T} \gamma\right)_{\nu}\right\}=\sum_{\rho, \sigma} \bar{a}_{\rho \mu} \bar{a}_{\sigma \nu}\left\{\gamma_{\rho}, \gamma_{\sigma}\right\}=2 \sum_{\rho} \bar{a}_{\rho \mu} \bar{a}_{\rho \nu}=2 \delta_{\mu \nu}
$$

Taking into account the form of the matrix $\bar{a}$ defined in Appendix A, $\bar{\gamma}$ is a gamma matrix if and only if the following two conditions hold

$$
\begin{aligned}
& \sum_{\rho} \bar{a}_{\rho \mu} \bar{a}_{\rho v}=0 \quad \text { for } \mu \neq v \Rightarrow \frac{3 S}{C}= \pm 1 \quad \Rightarrow \quad C=\frac{3}{\sqrt{10}}, \\
& \sum_{\rho} \bar{a}_{\rho \mu} \bar{a}_{\rho v}=1 \quad \text { for } \mu=v \Rightarrow R^{2}=3+\left(\frac{3 S}{C}\right)^{2} .
\end{aligned}
$$

Therefore, from this discussion, it can be concluded that the right continuum limit of the quark propagator can be obtained only when $C=3 / \sqrt{10}$ and $R=2$.

However, motivated by the first version of [7], we have decided to study as well the case $C=3 / \sqrt{14}$. In this case we know that $\bar{\gamma}$ is not a gamma matrix, since the condition given by Eq. (9) does not hold. $R$ can be still determined and must be $R=2 \sqrt{2}$ in order to satisfy Eq. (10). As expected, the analytical expression obtained for the quark propagator in this case does not correspond to the continuum one.

But, as it will be shown in the next sections where we present our results, the continuum limits for the pseudoscalar correlation function, mass and decay constant turn out to be the correct ones for the two values of $C$ studied here.

There are two reasons to explain this behaviour. On one hand, in the correlation function only the contribution from the pole survives in the sum over the momenta. This implies that all the possible crossed terms of the momentum which appear in the quark propagator, whose origin is the non-realization of Eq. (9), are cancelled. Moreover the properly chosen value of $R$ normalizes the quark propagator in order to obtain the 'would-be' correct continuum limit in absence of crossed terms.

\section{Boricifermions}

The action suggested by Borici [8] is a slight modification of the Creutz-Dirac operator and reads

$$
D_{B}(p)=\sum_{\mu} i \gamma_{\mu} \stackrel{\circ}{p}_{\mu}-i \frac{a}{2} \sum_{\mu} \Gamma_{\mu} \hat{p}_{\mu}^{2}+m_{0} \mathbb{1},
$$


where $\Gamma_{\mu}$ are linear combinations of gamma matrices defined in Appendix A. The corresponding propagator can be written as

$$
\tilde{S}_{B}(p)=\frac{-i \sum_{\mu} G_{\mu}(a p) \gamma_{\mu}+m_{0} \mathbb{1}}{\sum_{\mu} G_{\mu}(a p)^{2}+m_{0}^{2}},
$$

where the functions $G_{\mu}(a p)$, defined in the appendix, are trigonometric functions of their argument.

\section{Correlation functions}

In this section we give the expressions for the pseudoscalar and proton correlation functions at tree-level of perturbation theory. While we evaluated the pseudoscalar correlation function for all lattice fermions considered in this work, we use the proton correlation function only to demonstrate how the $\mathrm{O}(a)$ improvement works when the Wilson average or equivalently the mass average is performed in case standard Wilson fermions are considered.

The interpolating fields describing the charged pions, $\pi^{+}$and $\pi^{-}$are

$$
\mathcal{P}^{ \pm}(x) \equiv \mathcal{P}^{1}(x) \mp i \mathcal{P}^{2}(x)
$$

where $\mathcal{P}^{a}(x)=\bar{\psi}(x) \gamma_{5} \frac{\tau^{a}}{2} \psi(x)$, with $a=1,2,3$, is the pseudoscalar density and $\tau^{a}$ are the standard Pauli matrices. The computation for Wilson twisted mass is performed in the twisted basis, where the form of the local operator (13) stays unchanged [2].

The quark propagator can be decomposed in terms of the gamma matrices as

$$
\tilde{S}(p)=S_{U}(p) \mathbb{1}+\sum_{\mu} S_{\mu}(p) \gamma_{\mu}
$$

in the case of overlap and Creutz fermions, while for twisted mass fermions an additional term proportional to $\gamma_{5}$ is present

$$
\tilde{S}(p)=S_{U}(p) \mathbb{1}+\sum_{\mu} S_{\mu}(p) \gamma_{\mu}+S_{5}(p) \gamma_{5}
$$

With such a decomposition, the pseudoscalar correlation function can be written as ${ }^{1}$

$$
C(t)=\frac{N_{c} N_{d}}{L^{3} T^{2}} \sum_{p_{4}} \sum_{p_{4}^{\prime}} \sum_{\vec{p}} \sum_{\mu} e^{i\left(p_{4}-p_{4}^{\prime}\right) t} S_{\mu}\left(\vec{p}, p_{4}\right) S_{\mu}^{*}\left(\vec{p}, p_{4}^{\prime}\right),
$$

with $\mu=U, 1,2,3,4$ or $\mu=U, 1,2,3,4,5$ depending on the kind of fermion that is being considered. $N_{c}$ is the number of colours and $N_{d}$ is the number of Dirac components. $T=a N_{4}$ and $N_{4}$ is the number of lattice points in the time direction. The expression in Eq. (16) can be evaluated as it stands, or, a time-momentum representation of the quark propagator can be obtained for a lattice with infinite time extension, by performing the integration over $p_{4}$ analytically, see [13] for a discussion in case of twisted mass fermions. We have mostly used the representation of Eq. (16), but checked for twisted mass fermions the results also in the time-momentum representation. In Appendix A we give the explicit expression for the Wilson twisted mass quark propagator in the time-momentum representation for a lattice with infinite time extent.

\footnotetext{
1 The Wtm propagator has also a flavour structure. For the pseudoscalar correlation function only a single flavour component is needed.
} 
The local interpolating field describing the proton is given by ${ }^{2}$

$$
\mathcal{P}_{\alpha}(x) \equiv-\sqrt{2} \epsilon_{a b c}\left[\bar{d}_{a}^{T}(x) C^{-1} \gamma_{5} u_{b}(x)\right] u_{\alpha, c}(x) .
$$

The expression for the time dependence of the proton correlation function for standard Wilson fermions is then

$$
C_{\mathcal{P} \overline{\mathcal{P}}}(t)=\frac{N_{c} N_{d}}{L^{6}} \sum_{\vec{p}} \sum_{\vec{q}}\left\{L_{U}(\vec{p}, \vec{q}, t)+L_{4}(\vec{p}, \vec{q}, t)\right\},
$$

with the definitions

$$
\begin{aligned}
L_{U}(\vec{p}, \vec{q}, t) \equiv & S_{U}(-(\vec{p}+\vec{q}), t)\left\{\left(N_{d}+1\right) S_{U}(\vec{p}, t) S_{U}(\vec{q}, t)\right. \\
& \left.+\left(N_{d}+3\right) \sum_{\mu=1}^{4} S_{\mu}(\vec{p}, t) S_{\mu}(\vec{q}, t)\right\} \\
L_{\mu}(\vec{p}, \vec{q}, t) \equiv & S_{\mu}(-(\vec{p}+\vec{q}), t)\left\{\left(N_{d}+3\right) S_{U}(\vec{p}, t) S_{U}(\vec{q}, t)\right. \\
& \left.+\left(N_{d}+1\right) \sum_{\mu=1}^{4} S_{\mu}(\vec{p}, t) S_{\mu}(\vec{q}, t)\right\} .
\end{aligned}
$$

$S_{U}$ and $S_{\mu}$ are the components of the quark propagator for standard Wilson fermions, which can be obtained from the expression of the quark propagator for Wilson twisted mass fermions, given in Appendix A in Eq. (A.1), just by setting $\mu_{q}=0$. These results have been cross-checked with a standard inverter on a cold gauge configuration.

\section{Scaling tests on correlation functions, masses and decay constants}

In this section we give some results from a scaling analysis in the lattice spacing. At tree-level a dimensionless quantity can be only a function of $m L, a / L$ and $a m$, where $m$ here indicates generically the quark mass. To perform the continuum limit one can fix $m L$ to a certain value and the remaining dependence of the dimensionless quantity will be then in $a / L$. The continuum limit is then obtained sending $N=L / a$ to infinity. In the following we will set $a=1$ and the $1 / N$ and $1 / N^{2}$ dependence of the dimensionless quantities under investigation will correspond to $\mathrm{O}(a)$ and $\mathrm{O}\left(a^{2}\right)$ scaling violations. We remind that if not infinity the time extent $T$ will be always set to be proportional to $L$.

In particular, we consider the correlation function $C$ at a fixed physical time $t / N$, the pseudoscalar decay constant $f_{\mathrm{PS}}$ and the pseudoscalar and proton masses $M$. This leads us to consider the dimensionless quantities $N^{3} C(t / N), N M$ and $N f_{\mathrm{PS}}$.

We will start our discussion with an explicit demonstration of $\mathrm{O}(a)$ improvement for standard Wilson fermions from the Wilson and mass averaging procedures. We then turn over to the comparison of the size of scaling violations from all three lattice fermions considered here. In particular, we will compare the meson correlation function as well as the corresponding meson mass and meson decay constant.

\footnotetext{
2 The Greek (Latin) letters denote Dirac (colour) components and $u, d$ denote the flavour content. $C$ is the charge conjugation matrix and [ ] denotes spin trace.
} 

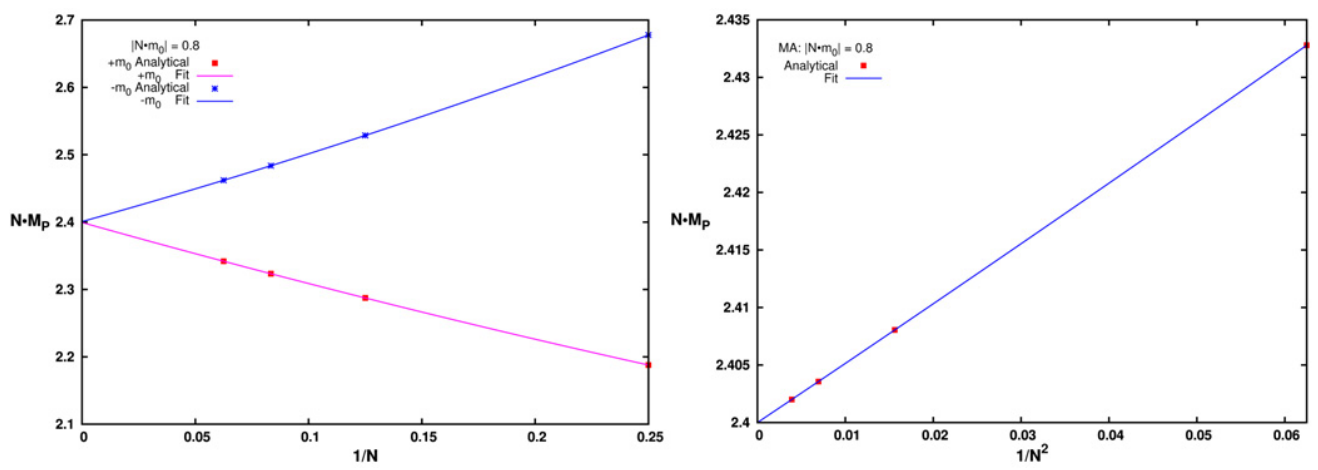

Fig. 1. In the left graph, the cutoff effects and the continuum limit of the proton mass obtained from two standard Wilson actions differing only in the sign of the quark mass, $\left|N m_{0}\right|=0.8$ are shown. The lattices are $4 \leqslant N \leqslant 20$. The lines are fits according to Eq. (21). In the right graph, the average of the proton masses obtained from the same two standard Wilson regularizations with quark masses $N m_{0}= \pm 0.8$ (MA) has been calculated. The solid line represents a fit to Eq. (21).

\subsection{Wilson average and mass average for standard Wilson fermions}

In Ref. [3] it has been demonstrated that when averaging physical observables computed with standard Wilson actions, i.e., setting $\mu_{q}=0$ in Eq. (1), having opposite signs of the quark mass $m_{0}$ (MA) or opposite signs of the Wilson parameter $r$ (WA), these quantities are $\mathrm{O}(a)$ improved. ${ }^{3}$ Since WA and MA at tree-level are equivalent, we will in the following only discuss the MA. As the physical observable we consider the proton mass as it can be obtained as the effective mass from the correlation function in Eq. (18) using timeslices at $t=4 N$ and $t=4 N+1$. For the computation we fix $\left|N m_{0}\right|=0.8$. In the left graph of Fig. 1 the behaviour of the proton mass $N M_{P}$ as a function of $\frac{1}{N}$ is given when two standard Wilson regularizations, differing only in the sign of the quark mass, are used.

The behaviour of the proton mass in Fig. 1 is linear in $1 / N$ showing the expected $\mathrm{O}(a)$ scaling violations of standard Wilson fermions. The proton mass reaches, in both cases, its continuum value of $N M_{P}=3\left|N m_{0}\right|$, as expected in the absence of interaction. However, the slopes with which the continuum value is reached, i.e., the coefficients of the $\mathrm{O}(a)$ cutoff effects differ in sign. The right graph of Fig. 1 shows the continuum approach of the proton mass when the proton mass is averaged over positive and negative values of the quark mass $N m_{0}$. In this case, the proton mass is plotted against $1 / N^{2}$ and the linearity in $1 / N^{2}$ nicely shows the $\mathrm{O}(a)$-improvement when the MA is applied.

In order to be more quantitative, we have used the following fitting functions to describe the analytically computed values of the proton mass:

$$
y_{1}=a_{0}+a_{1} \frac{1}{N}+a_{2} \frac{1}{N^{2}}, \quad y_{2}=b_{0}+b_{1} \frac{1}{N^{2}}+b_{2} \frac{1}{N^{4}} .
$$

Here $y_{1}\left(y_{2}\right)$ is the physical observable under consideration and its value in the continuum limit is given by the coefficient $a_{0}\left(b_{0}\right)$. We use two functional forms, the first formula of Eq. (21) for

\footnotetext{
3 The MA is actually done taking into account the chirality of the correlation function under investigation [3]. This is practically irrelevant for our study.
} 


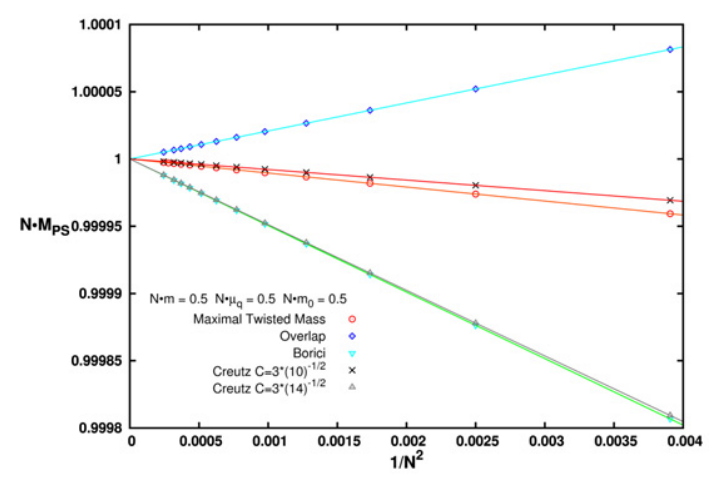

Fig. 2. The cutoff effects and the continuum limit of the pseudoscalar mass is shown. We compare overlap, maximally twisted mass and Creutz fermions at fixed quark mass, $N m=0.5, N \mu_{q}=0.5$ and $N m_{0}=0.5$, respectively. The lattices are $4 \leqslant N \leqslant 64$.

a leading $\frac{1}{N}$ behaviour (standard Wilson fermions) and the second formula for $\mathrm{O}(a)$-improved quantities.

The two lines in the left graph of Fig. 1 originate from a fit to Eq. (21) and correspond to the proton mass obtained from the same Wilson actions differing only in the sign of the quark mass. From the plot it is clear that in both cases the value of the proton mass in the continuum limit is the same and the expected one at tree-level of PT.

From the fit, the corresponding coefficients $a_{1}$ turn out to be the same in magnitude but have opposite signs for positive and negative quark masses. Thus, performing the (MA), it is to be expected that the $\mathrm{O}(a)$ effects cancel and the scaling behaviour changes from a $\frac{1}{N}$ to a $\frac{1}{N^{2}}$ behaviour. This can indeed be seen in the right graph of Fig. 1. Inspecting the fit coefficients $a_{2}$ and $b_{1}$, we find $a_{2} \approx b_{1} \approx 0.5$. Therefore, the magnitude of the leading order cutoff effects does not only change from an $\mathrm{O}(a)$ to an $\mathrm{O}\left(a^{2}\right)$ behaviour but also the $\mathrm{O}\left(a^{2}\right)$ effects do not increase when performing the Wilson average with respect to the standard case.

One interesting observation is the mass dependence of the coefficients $a_{1}$ and $b_{1}$ of Eqs. (21) for the pion and proton masses. We observe that in the case of standard Wilson fermions, the coefficient $a_{1} / a_{0}$, which determines the relative size of the $\mathrm{O}(a)$ cutoff effects, vanishes in the chiral limit proportionally to $N m_{0}$. For Wtm fermions at maximal twist the coefficient $b_{1} / b_{0}$, which determines the relative size of the $\mathrm{O}\left(a^{2}\right)$ cutoff effects, vanishes in the chiral limit, proportionally to $\left(N \mu_{q}\right)^{2}$.

\subsection{Comparing maximally twisted mass, overlap and Creutz fermions}

One interesting question is the relative size of cutoff effects when comparing maximally twisted mass, overlap and Creutz fermions. We have therefore performed a scaling analysis for the correlation functions themselves at a fixed physical distance, the pseudoscalar mass and the pseudoscalar decay constant. Since all the quantities under investigation are $\mathrm{O}(a)$-improved, we show them all as a function of $1 / N^{2}$.

Let us start with the pseudoscalar mass in Fig. 2. As expected, we indeed find a nice linear behaviour of the mass as a function of $1 / N^{2}$. However, we observe that the different kind of lattice fermions we have studied show quite distinct lattice artefacts at $\mathrm{O}\left(a^{2}\right)$. This can also be seen in Fig. 3, where we exhibit the dependence of the correlation function and the pseudoscalar decay constant as a function of $1 / N^{2}$. 

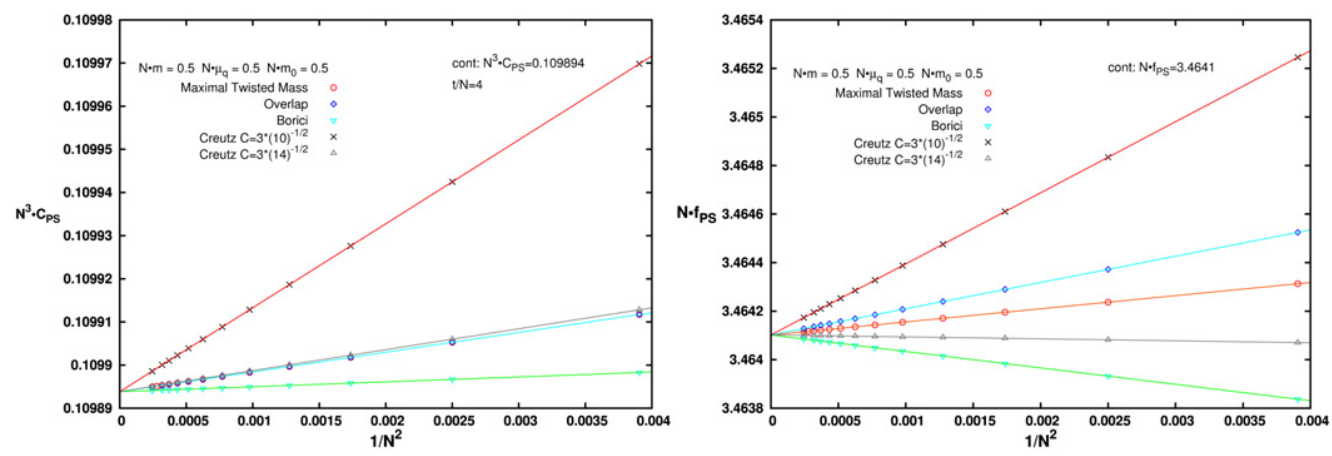

Fig. 3. In the left graph, the cutoff effects and the continuum limit of the pseudoscalar correlation function is shown. We compare overlap, maximally twisted mass and Creutz fermions at fixed quark mass, $N m=0.5, N \mu_{q}=0.5$ and $N m_{0}=0.5$, respectively. The lattices are $4 \leqslant N \leqslant 64$. In the right graph, the pseudoscalar decay constants obtained from the same lattice fermions as in the left graph are shown.

In order to see the cutoff effects in a quantitative way, the coefficients of the fit and the corresponding fit function for the three lattice quantities here considered, obtained from the different regularizations are presented in Tables 1, 2 and 3, respectively.

From the results obtained here, no clear picture of a particularly good or bad fermion discretization emerges. While we find that indeed all three kind of lattice fermions show the expected $\mathrm{O}(a)$-improvement, the (relative) size of the $\mathrm{O}\left(a^{2}\right)$ effects depends pretty much on the observable considered. If at all, one could say that maximally twisted mass fermions show uniformly small $\mathrm{O}\left(a^{2}\right)$ cutoff effects. On the other hand, it is somewhat amazing that Creutz fermions which break a number of important discrete symmetries do not suffer from very large $\mathrm{O}\left(a^{2}\right)$ cutoff effects. From our scaling analysis it is not possible to exclude a certain type of lattice fermion. Only scaling tests for the interacting theory will reveal the size of actual scaling violations of the observable considered.

\section{Effects from non-optimal tuning}

This section is devoted to the question of effects when tuning is performed non-optimally. In particular, we study the cutoff effects when there is an $\mathrm{O}(a)$ error in tuning to maximal twist. As a second example, we consider the case when the quark masses of two lattice fermion formulations are not exactly matched. This case is relevant for so-called mixed action simulations.

\subsection{Out of maximal twist}

Here we want to study a situation when we allow an $\mathrm{O}(a)$ error in setting the untwisted quark mass to zero. In order to realize this situation at tree-level of perturbation theory we 'force' these effects by simply fixing the twisted mass to be the physical quark mass and the untwisted mass is set to be proportional to $\frac{1}{N}$, as $N \mu_{q}=\alpha$ and $N m_{0}=\frac{\beta}{N} \backsim \mathrm{O}(a)$ where $\alpha$ is kept fixed and $\beta$ is a measure parametrizing the amount of violation of the maximal twist setup. The twist angle $\omega$ and the bare polar mass $M$ can be obtained as a function of $\alpha$ and $\beta$ as

$$
\omega=\frac{\pi}{2}-\left(\frac{\beta}{\alpha}\right) \frac{1}{N}+\mathrm{O}\left(\frac{1}{N^{2}}\right), \quad N M=\alpha\left[1+\frac{1}{2}\left(\frac{\beta}{\alpha}\right)^{2} \frac{1}{N^{2}}+\mathrm{O}\left(\frac{1}{N^{4}}\right)\right] .
$$


Table 1

Table of fit coefficients for the pseudoscalar mass using $N M_{\mathrm{PS}}=a+b \frac{1}{N^{2}}+c \frac{1}{N^{4}}$

\begin{tabular}{llcc}
\hline$N M_{\text {PS }}$ & $a$ & $b$ & $c$ \\
\hline MTM & 1 & -0.0104167 & 0.000292154 \\
Overlap & 1 & 0.0208333 & 0.000783943 \\
Borici & 1 & -0.0494786 & 0.00558893 \\
Creutz- $\sqrt{10}$ & 1 & -0.00781168 & -0.010171 \\
Creutz- $\sqrt{14}$ & 1 & -0.0488288 & 0.00287405 \\
\hline
\end{tabular}

Table 2

Table of fit coefficients for the pseudo scalar decay constant using $N f_{\mathrm{PS}}=a+b \frac{1}{N^{2}}+c \frac{1}{N^{4}}$

\begin{tabular}{llcc}
\hline$N f_{\text {PS }}$ & $a$ & $b$ & $c$ \\
\hline MTM & 3.4641 & 0.0541266 & -0.000815548 \\
Overlap & 3.4641 & 0.108253 & 0.00554908 \\
Borici & 3.4641 & -0.0676637 & -0.00486739 \\
Creutz- $\sqrt{10}$ & 3.4641 & 0.293217 & -0.0770494 \\
Creutz- $\sqrt{14}$ & 3.4641 & -0.00790885 & -0.0367598 \\
\hline
\end{tabular}

Table 3

Table of fit coefficient for the pseudo scalar correlation function using $N^{3} C=a+b \frac{1}{N^{2}}+c \frac{1}{N^{4}}$

\begin{tabular}{lccc}
\hline$N^{3} C(t / N=4)$ & $a$ & $b$ & $c$ \\
\hline MTM & 0.109894 & 0.00457891 & $-3.33302 \times 10^{-5}$ \\
Overlap & 0.109894 & 0.0045789 & 0.000181822 \\
Borici & 0.109894 & 0.00114427 & -0.00135812 \\
Creutz- $\sqrt{10}$ & 0.109894 & 0.0194625 & -0.00286602 \\
Creutz- $\sqrt{14}$ & 0.109894 & 0.00486428 & -0.002942 \\
\hline
\end{tabular}

Therefore, even if the condition of maximal twist can only be obtained up to $\mathrm{O}(a)$ cutoff effects, which is generally the case in practical numerical simulations, the observables, which are only functions of the polar mass, are still automatically $\mathrm{O}(a)$ improved.

Moreover, Eq. (22) also shows how the size of the leading discretization effects depends on the ratio between the untwisted and twisted quark masses. This ratio in turn determines the value of the lattice spacing at which the asymptotic $\frac{1}{N^{2}}$ scaling sets in. Only when this ratio is small enough and hence the lattice does not need to be chosen too large a reliable continuum limit using reasonably sized lattices can be performed. The left graph of Fig. 4 demonstrates that the asymptotic scaling sets in for lattices with $4 \leqslant N \leqslant 64$ when $0 \lesssim \frac{\beta}{\alpha} \lesssim 2$.

However, for $\frac{\beta}{\alpha} \gtrsim 10$ the continuum limit is not reliable anymore if $N$ is chosen to be too small. This can be seen in the right graph of Fig. 4. Using only small values of $N$ leads to an inconsistent continuum limit value. Therefore, larger lattices are needed in order to obtain the correct continuum behaviour as can be also seen in the right graph of Fig. 4. Here we have added a fit of the data for a value of $\beta=10.0$ taking only large lattices into account, i.e., using only values of $N \geqslant 40$. In this case, indeed the right continuum value is obtained. Of course, for practical simulations, using only lattices with $N \gg 40$ appears to be rather unrealistic so to chose a correct ratio $\frac{\beta}{\alpha}$ becomes important. 

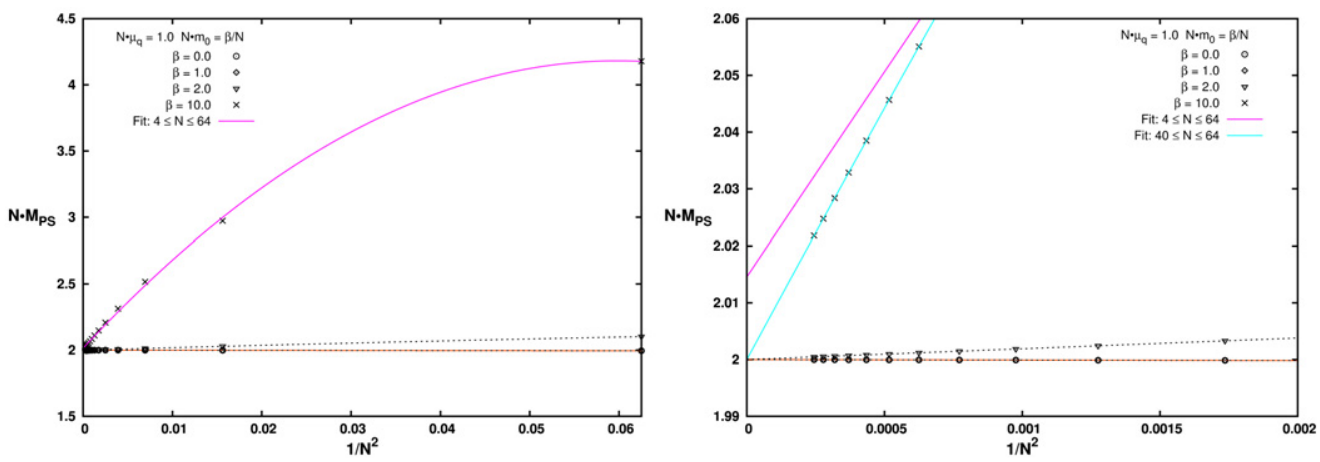

Fig. 4. Left graph: Behaviour of the pion mass as a function of $\frac{1}{N^{2}}$, for lattices with size $4 \leqslant N \leqslant 64$. The twisted quark mass is set to $N \mu_{q}=1.0$ and the untwisted quark mass is zero up to $\mathrm{O}(a)$ cutoff effects, i.e., $N m_{0}=\frac{\beta}{N}$ with $\beta=0.0,1.0,2.0,10.0$. Right graph: a zoom of the graph on the left with an additional fit for the analytical data corresponding to $\beta=10.0$ which considers only large lattices $40 \leqslant N \leqslant 64$.

\subsection{Unmatched quark masses}

In this section we want to study the continuum limit and the size of the cutoff effects of lattice quantities constructed from ratios of physical observables computed on the lattice from two different regularizations, i.e., here Wilson twisted mass fermions at maximal twist and overlap fermions. In particular, we want to study the situation when both quark masses are not exactly fixed to the same value but differ up to $\mathrm{O}\left(a^{2}\right)$ effects.

The reason for studying such setup is that in real simulations using a mixed but $\mathrm{O}(a)$-improved action both masses can be fixed to the same value only up to $\mathrm{O}\left(a^{2}\right)$ effects. In order to realize non-matched quark masses, we fix the twisted quark mass exactly at $N \mu_{q}=0.5$ and allow for an $\mathrm{O}\left(a^{2}\right)$ error in setting the overlap quark mass,

$$
N m=0.5-v / N^{2} \text {. }
$$

We will vary the parameter $v$ from $v=0$ to $v=4.0$.

Similar to the case of twisted mass at a non-optimally tuned value of the bare quark mass, the leading $\mathrm{O}\left(a^{2}\right)$ cutoff effects can become very large when the quark masses are substantially mis-matched. This might even induce the danger of not achieving the right continuum limit when only too small lattice sizes are used.

This is exemplified in Fig. 5. In the left graph we show the scaling behaviour in $1 / N^{2}$ for various values of the parameter $v$ for the full range of the lattice sizes. If $v$ is small, we observe $1 / N^{2}$ scaling violations. Only in the case $v=4$, do the $1 / N^{4}$ corrections become relevant. More quantitatively a linear fit in $1 / N^{2}$ including all the data points starting from $N=4$ is reliable for $v \lesssim 1$. While for $v \simeq 4$ we can include, in a linear fit in $1 / N^{2}$, only the data points starting from $N=12$.

Fig. 5 demonstrates that not only the slope of the ratio of pseudoscalar masses versus $1 / N^{2}$ increases as we make the value of $v$ larger, but also that the $\mathrm{O}\left(a^{4}\right)$ effects become significant as can be seen from the curvature in the plot for $v=4$. Moreover, for $v=4$, the continuum limit fit is not reliable anymore if too small lattices are considered, which can be seen in the right graph of Fig. 5. Thus, if the matching is not performed with a good enough precision, large lattices have to be used to avoid higher order cutoff effects and perform a reliable continuum limit. 

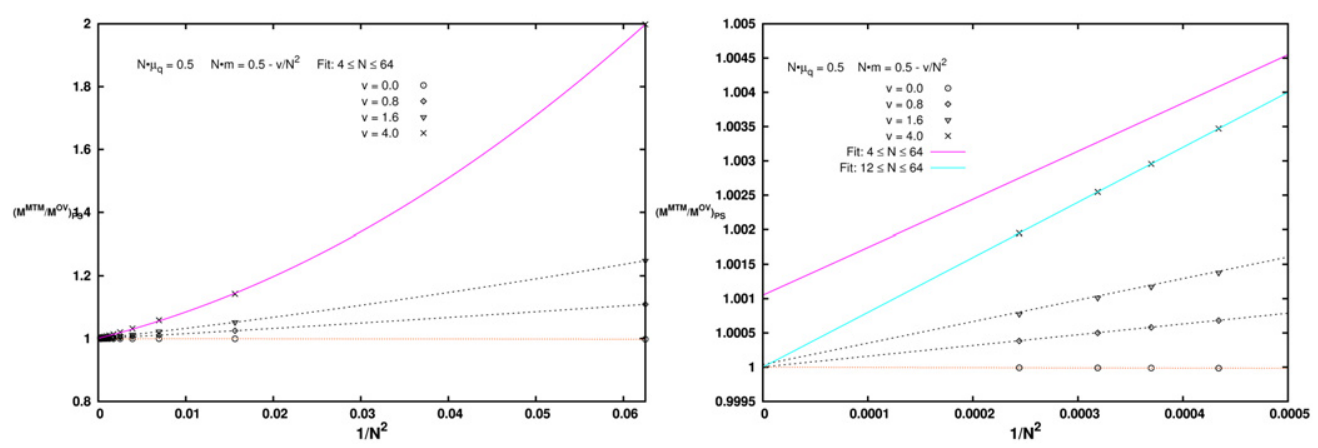

Fig. 5. The cutoff effects and continuum limit of the ratio of the pseudoscalar mass computed for maximally twisted mass and overlap fermions. In both graphs $N \mu_{q}=0.5, N m=0.5-0.4 / N^{2}$ and $t / N=4$. The left graph shows the full range of lattice sizes considered while the right graph represents a zoom.

\section{Conclusions}

In this paper we have performed a scaling test in the lattice spacing towards the continuum limit for three kinds of lattice fermions. Our setup has been tree-level of perturbation theory. The lattice fermions considered here were Wilson twisted mass, overlap and Creutz and Borici fermions. We looked at the pseudoscalar correlation function at a fixed time and the corresponding pseudoscalar mass and decay constant.

As a first step, we have verified automatic $\mathrm{O}(a)$ improvement for Wilson twisted mass fermions and showed, with the example of the proton mass, the mechanisms of mass average.

The relative comparison of all three kinds of lattice fermions we have considered did not result in a clear picture in the sense that one lattice fermion shows consistently smaller or bigger $\mathrm{O}\left(a^{2}\right)$ lattice artefacts than the other. Rather we found that the sizes of $\mathrm{O}\left(a^{2}\right)$ lattice artefacts depend on the observable considered with perhaps the exception of maximally twisted mass fermions which shows a rather uniform behaviour with small $\mathrm{O}\left(a^{2}\right)$ effects. Therefore, we expect that also in practical simulations the $\mathrm{O}\left(a^{2}\right)$ lattice artefacts can turn out to be quite different depending on the physical observable considered.

Finally, we studied the situation when parameters are tuned non-optimally. We considered twisted mass fermions when an $\mathrm{O}(a)$ error was allowed in the tuning to maximal twist. In addition, we looked at ratios of physical quantities built from overlap and twisted mass fermions. In this case we allowed an $\mathrm{O}\left(a^{2}\right)$ error in the matching of the quark masses. Our conclusion of these studies is that when the corresponding $\mathrm{O}\left(a^{2}\right)$ error is too large, the continuum limit becomes not reliable if the lattice spacing is not small enough.

\section{Acknowledgements}

We thank M. Brinet, V. Drach and C. Urbach for numerical checks of the Wilson twisted mass results presented here. We thank Dru Renner for careful reading of this paper and useful comments. A.S. thanks Chris Michael for discussions about Creutz fermions. A.S. acknowledges, for providing hospitality, DESY-Zeuthen where a big part of this work has been done. J.G.L. thanks M. Müller-Preussker for discussions. This work has been supported in part by the DFG Sonderforschungsbereich/Transregio SFB/TR9-03. 


\section{Appendix A}

In this appendix we summarize some of the formulæ and definitions which have been used in this work.

\section{Wilson twisted mass fermions}

For Wilson twisted mass fermions we give the analytic expression for the time-momentum representation of the quark propagator on a lattice with infinite time extent. Details of the computations can be found in Ref. [13].

The propagator reads, ${ }^{4}$

$$
\begin{aligned}
S(\vec{p}, t)= & \frac{1}{2 \mathcal{E}_{1}}\left\{\operatorname{sgn}(t) \sinh E_{1} \gamma_{4} \mathbb{1}_{f}-i a \mathcal{K}(\vec{p}) \mathbb{1}_{f}+\left[\left(1-\cosh E_{1}\right)+a M(\vec{p})\right] \mathbb{1}_{f}\right. \\
& \left.-i a \mu_{q} \gamma_{5} \tau^{3}\right\} e^{-E_{1}\left|\frac{t}{a}\right|}+\delta_{\frac{t}{a}, 0} \frac{1}{2(1+a M(\vec{p}))} \mathbb{1}_{f}
\end{aligned}
$$

with $^{5}$

$$
\begin{aligned}
& M(\vec{p})=m_{0}+\frac{a}{2} \sum_{i=1}^{3} \hat{p}_{i}^{2}, \quad \mathcal{K}(\vec{p})=\sum_{i=1}^{3} \gamma_{i} \stackrel{\circ}{p}_{i}, \\
& \cosh E_{1}=1+\frac{a^{2} \mathcal{K}^{2}(\vec{p})+a^{2} M^{2}(\vec{p})+a^{2} \mu_{q}^{2}}{2(1+a M(\vec{p}))}, \\
& \mathcal{E}_{1} \equiv|1+a M(\vec{p})| \sinh E_{1} .
\end{aligned}
$$

\section{Creutzfermions}

Our starting point for the analysis of the Creutz action is the operator given by

$$
D_{C}(p)=i \sum_{\mu}\left(\bar{s}_{\mu}(a p)+\bar{c}_{\mu}(a p)\right) \gamma_{\mu}+m_{0} \mathbb{1}
$$

where $\mathbb{1}$ is the identity matrix in Dirac space and the momentum has already been reexpressed in terms of the pole as

$$
q_{\mu}=\tilde{q}+p_{\mu} .
$$

Starting from a reciprocal lattice labelled here by $q_{\mu}$ the poles of the Creutz-Dirac operator are localized at $(\tilde{q}, \tilde{q}, \tilde{q}, \tilde{q})$ and $(-\tilde{q},-\tilde{q},-\tilde{q},-\tilde{q})$, where $\tilde{q}$ is related to the parameter $C$ by the equation $C=\cos (\tilde{q})$. In the following, we consider only the former pole. In addition, we also define the parameter $S=\sin (\tilde{q})$.

The relevant trigonometric functions for Creutz fermions are defined by

$$
\bar{s}_{k}(a p)=\frac{1}{R} s_{k}(a p), \quad \bar{s}_{4}(a p)=\frac{3 S}{R C} s_{4}(a p),
$$

\footnotetext{
4 This expression is valid for all the possible values of the discrete Euclidean time $t$, even if it is negative or zero. This generalization has been done by using the properties of the Fourier transform under change of sign of the argument.

$5 \operatorname{sgn}(t)$ is the sign of $t$, and we have denoted $\operatorname{sgn}(0) \equiv 0$. It is just a convention in order to give one general expression for the propagator for all possible values of $t$.
} 


$$
\bar{c}_{k}(a p)=\frac{S}{R C} c_{k}(a p), \quad \bar{c}_{4}(a p)=\frac{3}{R} c_{4}(a p),
$$

with the functions $s$ and $c$ given by

$$
\begin{aligned}
& s_{1}(\text { ap })=\left[\stackrel{\circ}{1}_{1}+\stackrel{\circ}{p}_{2}-\stackrel{\circ}{p}_{3}-\stackrel{\circ}{p}_{4}\right], \\
& s_{2}(\text { ap })=\left[\stackrel{\circ}{1}_{1}-\stackrel{\circ}{p}_{2}-\stackrel{\circ}{p}_{3}+\stackrel{\circ}{p}_{4}\right], \\
& s_{3}(\text { ap })=\left[\stackrel{\circ}{1}_{1}-\stackrel{\circ}{p}_{2}+\stackrel{\circ}{p}_{3}-\stackrel{\circ}{p}_{4}\right], \\
& s_{4}(\text { ap })=\left[-\stackrel{\circ}{1}_{1}-\stackrel{\circ}{2}_{2}-\stackrel{\circ}{p}_{3}-\stackrel{\circ}{p}_{4}\right], \\
& c_{1}(\text { ap })=-\frac{a}{2}\left[\hat{p}_{1}^{2}+\hat{p}_{2}^{2}-\hat{p}_{3}^{2}-\hat{p}_{4}^{2}\right], \\
& c_{2}(\text { ap })=-\frac{a}{2}\left[\hat{p}_{1}^{2}-\hat{p}_{2}^{2}-\hat{p}_{3}^{2}+\hat{p}_{4}^{2}\right], \\
& c_{3}(\text { ap })=-\frac{a}{2}\left[\hat{p}_{1}^{2}-\hat{p}_{2}^{2}+\hat{p}_{3}^{2}-\hat{p}_{4}^{2}\right], \\
& c_{4}(\text { ap })=-\frac{a}{2}\left[\hat{p}_{1}^{2}+\hat{p}_{2}^{2}+\hat{p}_{3}^{2}+\hat{p}_{4}^{2}\right] .
\end{aligned}
$$

With the expression of Eq. (A.5) we obtain the corresponding propagator with standard manipulations as

$$
\tilde{S}_{C}(p)=\frac{-i \sum_{\mu}\left(\bar{s}_{\mu}(a p)+\bar{c}_{\mu}(a p)\right) \gamma_{\mu}+m_{0} \mathbb{1}}{\sum_{\mu}\left(\bar{s}_{\mu}(a p)+\bar{c}_{\mu}(a p)\right)^{2}+m_{0}^{2}} .
$$

In order to understand the continuum limit of the Creutz-Dirac operator and the role of the factor $R$, we will use in the following the notation of Borici in [8], with a scalar product $(\gamma, x) \equiv$ $\sum_{\mu} \gamma_{\mu} x_{\mu}$

We thus write Eq. (A.5) as

$$
D_{C}(p)=i(\gamma, \bar{a} \tilde{s}(a p)+\bar{b} \tilde{c}(a p))+m_{0} \mathbb{1}=i\left(\bar{a}^{T} \gamma, \tilde{s}(a p)+\bar{a}^{-1} \bar{b} \tilde{c}(a p)\right)+m_{0} \mathbb{1},
$$

where

$$
\tilde{s}(\text { ap })=\frac{1}{a}\left(\stackrel{\circ}{p}_{1}, \stackrel{\circ}{p}_{2}, \stackrel{\circ}{p}_{3}, \stackrel{\circ}{p}_{4}\right)^{T}, \quad \tilde{c}(\text { ap })=-\frac{a}{2}\left(\hat{p}_{1}^{2}, \hat{p}_{2}^{2}, \hat{p}_{3}^{2}, \hat{p}_{4}^{2}\right)^{T}
$$

and the matrices $\bar{a}$ and $\bar{b}$

$$
\bar{a}=\frac{1}{R}\left(\begin{array}{cccc}
1 & 1 & -1 & -1 \\
1 & -1 & -1 & 1 \\
1 & -1 & 1 & -1 \\
-\frac{3 S}{C} & -\frac{3 S}{C} & -\frac{3 S}{C} & -\frac{3 S}{C}
\end{array}\right), \quad \bar{b}=\frac{1}{R} \frac{S}{C}\left(\begin{array}{cccc}
1 & 1 & -1 & -1 \\
1 & -1 & -1 & 1 \\
1 & -1 & 1 & -1 \\
\frac{3 C}{S} & \frac{3 C}{S} & \frac{3 C}{S} & \frac{3 C}{S}
\end{array}\right) .
$$

As a next step, we define

$$
\bar{\alpha} \equiv \bar{a}^{-1} \bar{b}, \quad \bar{\gamma} \equiv \bar{a}^{T} \gamma, \quad \bar{\Gamma} \equiv \bar{\alpha} \bar{\gamma}=\bar{\alpha} \bar{a}^{T} \gamma
$$

from which we obtain the final form of the Creutz-Dirac operator

$$
D_{C}(p)=i \sum_{\mu} \stackrel{\circ}{p}_{\mu} \bar{\gamma}_{\mu}-i \frac{a}{2} \sum_{\mu} \hat{p}_{\mu}^{2} \bar{\Gamma}_{\mu}+m_{0} \mathbb{1} .
$$

This is the expression given in Eq. (6). 


\section{Boricifermions}

Here we give the definitions we have used for Borici fermions. The $\Gamma$ matrices multiplying the Wilson term are defined by

$$
\Gamma_{\mu}=\sum_{\nu} \alpha_{\mu \nu} \gamma_{\nu}
$$

with the definition

$$
\alpha=\frac{1}{2}\left(\begin{array}{cccc}
1 & -1 & -1 & -1 \\
-1 & 1 & -1 & -1 \\
-1 & -1 & 1 & -1 \\
-1 & -1 & -1 & 1
\end{array}\right) .
$$

We incidentally note that $\sum_{\mu} \gamma_{\mu}=-\sum_{\mu} \Gamma_{\mu}$.

The trigonometric functions appearing in the Borici propagator are given by

$$
\begin{aligned}
& G_{1}(\text { ap })=\stackrel{\circ}{p}_{1}-\frac{a}{4}\left[\hat{p}_{1}^{2}+\hat{p}_{2}^{2}-\hat{p}_{3}^{2}-\hat{p}_{4}^{2}\right], \\
& G_{2}(a p)=\stackrel{\circ}{2}_{2}-\frac{a}{4}\left[-\hat{p}_{1}^{2}+\hat{p}_{2}^{2}-\hat{p}_{3}^{2}-\hat{p}_{4}^{2}\right], \\
& G_{3}(a p)=\stackrel{\circ}{p}_{3}-\frac{a}{4}\left[-\hat{p}_{1}^{2}-\hat{p}_{2}^{2}+\hat{p}_{3}^{2}-\hat{p}_{4}^{2}\right], \\
& G_{4}(a p)=\stackrel{\circ}{p}_{4}-\frac{a}{4}\left[-\hat{p}_{1}^{2}-\hat{p}_{2}^{2}-\hat{p}_{3}^{2}+\hat{p}_{4}^{2}\right] .
\end{aligned}
$$

\section{References}

[1] K. Cichy, et al., arXiv: 0710.2036 [hep-lat].

[2] ALPHA Collaboration, R. Frezzotti, et al., JHEP 0108 (2001) 058, hep-lat/0101001.

[3] R. Frezzotti, G.C. Rossi, JHEP 0408 (2004) 007, hep-lat/0306014.

[4] A. Shindler, arXiv: 0707.4093 [hep-lat].

[5] H. Neuberger, Phys. Lett. B 417 (1998) 141, hep-lat/9707022.

[6] F. Niedermayer, Nucl. Phys. B (Proc. Suppl.) 73 (1999) 105, hep-lat/9810026.

[7] M. Creutz, arXiv: 0712.1201 [hep-lat].

[8] A. Borici, arXiv: 0712.4401 [hep-lat].

[9] P.F. Bedaque, et al., arXiv: 0801.3361 [hep-lat].

[10] M. Lüscher, Phys. Lett. B 428 (1998) 342, hep-lat/9802011.

[11] P. Hernandez, K. Jansen, M. Lüscher, Nucl. Phys. B 552 (1999) 363, hep-lat/9808010.

[12] D.B. Carpenter, C.F. Baillie, Nucl. Phys. B 260 (1985) 103.

[13] J. González López, Cutoff effects for Wilson twisted mass fermions at tree-level of perturbation theory, 2007.

[14] $\chi \mathrm{E}$ Collaboration, W. Bietenholz, et al., JHEP 0412 (2004) 044, hep-lat/0411001. 